\title{
Lunar Soils: Phase Equilibria and Transport Properties
}

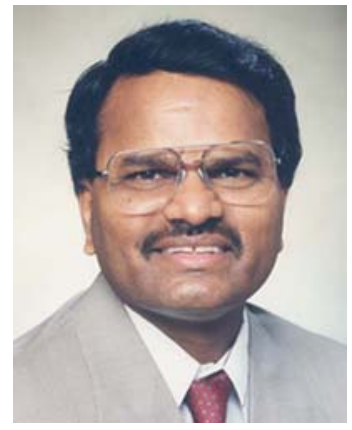

Journal of Phase Equilibria and Diffusion (JPED) has been providing valuable information for various materials systems to academia, research and industry communities. The basic and applied researches on processing in a variety of materials systems that are known on earth are well studied. But very little or no phase equilibria and transport data is available for lunar soils.

NASA's exploration and space operations programs and the project-In-Situ Resource Utilization (ISRU), in an effort towards establishing a self-sufficient planetary habitat, will invariably require materials for power generation ( $\mathrm{Si}$ for solar cells) and habitat construction (e.g. Al, Fe, and Ti). Investigations are needed to enable technology development that will lead to extraction and purification of these elements from in situ planetary resources in support of space missions beyond low-earth orbit (LEO). Therefore, such technological advances here on earth and on Lunar or Martian outpost would be in agreement with NASA's vision statement: 'to improve life here, to extend life to there, to find life beyond'.

The ultimate success of space exploration and colonization of the Moon will depend on the effective use of the available resources. Minerals and soils available on the Moon are excellent sources for extraction of oxygen, water, metals and alloys needed for extended human presence on the Moon. Processing of lunar regolith into usable structural components to support the construction of advanced Lunar bases and colonies will be essential for human habitation. It is economical to use locally produced metals and alloys. The primary problem that requires to be addressed is developing an energy efficient process for extraction of useful structural metals such as $\mathrm{Fe}, \mathrm{Si}, \mathrm{Al}$, and $\mathrm{Ti}$ from planetary resources available on the Moon.

Currently, most extraction processes being investigated are based on carbothermal reduction of simulant lunar soils using hydrogen, carbon monoxide, or methane. There are investigations on indirect electrochemical reduction processes for simulant lunar oxides using lithium in an electrolytic cell operating at about $900{ }^{\circ} \mathrm{C}$. Another technique that has received attention is the pyrolisis process where intense heat is used to decompose metal oxides at temperatures ranging between $1500-3000{ }^{\circ} \mathrm{C}$ followed by condensation. Despite the relative low level of success, the aforementioned techniques operate at temperatures higher than $1000{ }^{\circ} \mathrm{C}$. This demands high-energy consumption and limits the overall lifetime of the reactors.

Calculations of thermodynamic equilibrium concentrations of metallic species produced by carbothermal reduction of simulant lunar materials (JSC-1) were performed. The composition of reduced species from JSC-1 was calculated as a function of temperature and pressure. The results showed formation of metallic alloys under the reducing carbon and hydrogen atmospheres. The experimental results obtained from a carbothermally reduced JSC-1 samples showed the precipitation of the shiny alloy phases as reaction products. The average composition of alloys formed is in very good agreement with the thermodynamic model predictions.

The amount of hydrogen in the lunar system drastically affects the process of iron extraction. The yields of metallic iron from the lunar soil as function of temperature and hydrogen content were evaluated. Iron is the only metal that can be significantly extracted from the lunar soil by hydrogen. Even at a low temperature, metallic iron is still possible to exist on the surface of the Moon only if the concentration of hydrogen is higher than $0.0018 \%$ (wt.\%) in lunar soils. These results are in good agreement with metallic iron content observed in the Apollo 11 lunar soils obtained from the Moon.

Recently developed ionic liquids (ILs) are new promising electrolytes for electrolysis of metals and alloys at low temperatures. This technology has potential to be used for the extraction of metals from lunar soils. Studies have shown successful production of $\mathrm{Al}$ and alloys using ionic liquids. Due to its near room temperature processing environment, the new technology will overcome many of the limitations associated with high temperature processes such as high-energy consumption and corrosion. Further, ionic liquids are non-toxic and could be recycled after purification, thus minimizing extraction reagent losses and the earth supplied consumables.

Newer understanding of the thermodynamics and kinetics of material processing in the planetary surface environment are needed. Such process modules would meet the challenges for extended human presence on the Moon. Successful understanding of thermodynamics and transport properties of lunar materials would pave way for outerspace exploration and human habitation on the Moon.

In summary, relatively little research efforts have been made towards the fundamental understanding of the phase equilibria and transport properties of lunar soils. With the above mentioned opportunities and challenges, efforts are needed to generate thermodynamic and transport properties data of lunar soils. This will enhance our understanding of lunar soils for the development of planetary technologies. 